\title{
Fermentable fibres condition colon microbiota and promote diabetogenesis in NOD mice
}

\author{
Raine K. Toivonen • Rohini Emani • Eveliina Munukka • Anniina Rintala • Asta Laiho • \\ Sami Pietilä • Juha-Pekka Pursiheimo • Pasi Soidinsalo • Mari Linhala • Erkki Eerola • \\ Pentti Huovinen • Arno Hänninen
}

Received: 7 March 2014 / Accepted: 17 June 2014 / Published online: 17 July 2014

(C) Springer-Verlag Berlin Heidelberg 2014

\begin{abstract}
Aims/hypothesis Gut microbiota (GM) and diet both appear to be important in the pathogenesis of type 1 diabetes. Fermentable fibres (FFs), of which there is an ample supply in natural, diabetes-promoting diets, are used by GM as a source of energy. Our aim was to determine whether FFs modify GM and diabetes incidence in the NOD mouse.

Methods Female NOD mice were weaned to a semisynthetic diet and the effects of FF supplementation on diabetes incidence and insulitis were evaluated. Real-time quantitative PCR was employed to determine the effects imposed to gene transcripts in the colon and lymph nodes. Changes to GM were analysed by next-generation sequencing.

Results NOD mice fed semisynthetic diets free from FFs were largely protected from diabetes while semisynthetic diets supplemented with the FFs pectin and xylan (PX) resulted in higher diabetes incidence. Semisynthetic diet free from FFs altered GM composition significantly; addition of PX changed the composition of the GM towards that found in natural-dietfed mice and increased production of FF-derived short-chain fatty acid metabolites in the colon. The highly diabetogenic natural diet was associated with expression of proinflammatory and stress-related genes in the colon, while the
\end{abstract}

Electronic supplementary material The online version of this article (doi:10.1007/s00125-014-3325-6) contains peer-reviewed but unedited supplementary material, which is available to authorised users.

R. K. Toivonen $(\bowtie) \cdot$ R. Emani $\cdot$ E. Munukka $\cdot$ A. Rintala

M. Linhala $\cdot$ E. Eerola $\cdot$ P. Huovinen $\cdot$ A. Hänninen $(\bowtie)$

Department of Medical Microbiology and Immunology, University

of Turku, Kiinamyllynkatu 13, 20520 Turku, Finland

e-mail: raine.toivonen@utu.fi

e-mail: arno.hanninen@utu.fi

A. Laiho $\cdot$ S. Pietilä $\cdot$ J.-P. Pursiheimo $\cdot$ P. Soidinsalo

Turku Centre for Biotechnology, Tykistökatu 6,

20520 Turku, Finland semisynthetic diet free from FFs promoted Il4, Il22, Tgf $\beta$ and Foxp 3 transcripts in the colon and/or pancreatic lymph node. PX in the same diet counteracted these effects and promoted stress-related IL-18 activation in gut epithelial cells. 16S RNA sequencing revealed each diet to give rise to its particular GM composition, with different Firmicutes to Bacteroidetes ratios, and enrichment of mucin-degrading Ruminococcaceae following diabetes-protective FF-free diet. Conclusions/interpretation FFs condition microbiota, affect colon homeostasis and are important components of natural, diabetes-promoting diets in NOD mice.

Keywords Dietary intervention - Gut immune system . Microbiota - Type 1 diabetes

$\begin{array}{ll}\text { Abbreviations } \\ \text { BiP } & \text { Binding immunoglobulin protein } \\ \text { CE } & \text { Cellulose containing diet } \\ \text { CHOP } & \text { C/EBP homologous protein } \\ \text { FF } & \text { Fermentable fibre } \\ \text { GM } & \text { Gut microbiota } \\ \text { MLN } & \text { Mesenteric lymph node } \\ \text { NC } & \text { Normal rodent chow } \\ \text { PaLN } & \text { Pancreatic lymph node } \\ \text { PX } & \text { Pectin- and xylan-containing diet } \\ \text { SCFA } & \text { Short-chain fatty acid } \\ \text { Treg } & \text { Regulatory T cell }\end{array}$

\section{Introduction}

The NOD mouse spontaneously develops autoimmune diabetes, which closely resembles human type 1 diabetes (reviewed in $[1,2])$. Current evidence suggests a close relationship between development of type 1 diabetes and the gut immune 
system [3-5]. Inflammatory cells and markers in intestinal biopsies, increased levels of antibodies to food antigens in children who develop type 1 diabetes, shared determinants of leucocyte traffic between gut and pancreas $[6,7]$ and increased permeability of the gut $[8,9]$ all provide evidence supporting such a relationship. The importance of dietary factors in type 1 diabetes development is directly proven in animal models of autoimmune diabetes [10-12].

Studies in rodent models of autoimmune diabetes, as well as in humans, also suggest that the composition of gut microbiota (GM) is an important determinant of islet autoimmunity and type 1 diabetes [13]. Disruption of immune homeostasis in the colon by chemical irritation or Citrobacter rodentium infection promotes activation of islet-reactive $\mathrm{T}$ cells in the pancreatic lymph node (PaLN) [14, 15]. In NOD mice, anti-diabetogenic infant formula diet both alleviates inflammatory phenomena in the gut and affects microbiota composition in the large intestine $[12,16]$, suggesting that the immune mechanisms evoked by commensal microbes in the large intestine associate with autoimmune diabetes.

Studies in the BB rat and NOD mouse indicate that natural cereal-based rodent diets are the most diabetogenic, while hypoallergenic infant formulae consisting of hydrolysed peptides or soy protein are associated with protection against diabetes. While most dietary factors, including proteins, are digested and absorbed in the small intestine by host-dependent mechanisms, dietary fibres pass to the large intestine intact and form an important part of luminal gut contents there. Certain gut bacteria are capable of hydrolysing these complex polysaccharides for their energy supply. While cellulose is hydrolysed inefficiently, pectin and xylan are hydrolysed more readily and thus may be called fermentable fibres (FFs). Hydrolysis of FFs results in the production of shortchain fatty acids (SCFAs), such as butyrate, which support epithelial cells and the integrity of immune homeostasis in the large intestine [17, 18]. Interestingly, some complex polysaccharides also irritate the large intestine $[19,20]$ and can disturb gut homeostasis with potential relevance to islet autoimmunity [14].

Due to the proposed roles of GM in type 1 diabetes and their effects on the gut immune system, we set forth to investigate whether the FFs pectin and xylan, present in natural diabetes-promoting diets and used by GM as energy source, have a role in diabetogenesis in the NOD mouse. Our data suggest that the lack of these compounds in semisynthetic diets protects NOD mice effectively from diabetes. This protection is associated with several potentially important phenomena, including a change in the composition of GM, reduction in stress-related transcripts $\mathrm{C} / \mathrm{EBP}$ homologous protein $(\mathrm{CHOP})$ and binding immunoglobulin protein $(\mathrm{BiP})$ and promotion of anti-inflammatory transcripts in the gut and PaLN and reduced IL-18 production in gut epithelial cells.

\section{Methods}

Animals Female NOD mice (NOD/ShiLtJ; Jackson Laboratories, Bar Harbor, ME, USA) were bred and housed in Turku University animal facility under specific-pathogen-free conditions and caged under filter covers. For a brief description of conditions see ESM Methods. All animal work was carried out by trained scientists with permission from the Finnish Laboratory Animal Board (license number: ESAVI/3210/ 04.10.03/2011). Mice from several litters were allocated into groups fed different diets from weaning. For complete semisynthetic diets, Basal mix 80\% (Harlan Laboratories, Madison, WI, USA) was used for base and was supplemented with $20 \%$ whey and casein and fibres (pectin and xylan or cellulose alone) as indicated (ESM Table 1). The blood glucose level was monitored weekly from 12 to 34 weeks of age by puncturing the tail vein. Blood glucose exceeding $14 \mathrm{mmol} / \mathrm{l}$ for 2 consecutive days was deemed diabetic. Samples were collected for colon epithelial cell isolation and cytokine profiling (colon, lymph nodes) after diets had been consumed for 4 weeks $(n=7)$. Insulitis was quantified by evaluating the degree of infiltration and categorised as follows: 0 , no insulitis; 1, peri-insulitis with or without minimal infiltration in islets; 2 , insulitis with $<50 \%$ infiltration of islets; 3 , invasive insulitis with $>50 \%$ infiltration of islets. Insulitis was determined from non-consecutive cryosections of pancreases after hematoxylin and eosin (H\&E) staining. At least 60 individual islets were graded for the level of infiltrating cells. Colon samples were fixed in paraformaldehyde and embedded in paraffin and sections were stained with H\&E staining for evaluation of colon mucosa thickness.

$R N A$ isolation and quantitative PCR To analyse diet-derived changes in cytokines and transcription factors, colon and lymph nodes were collected into RNAlater (Qiagen, Germantown, MD, USA). Total RNA was extracted with bead-based PowerLyzer RNA Isolation Kit (MoBio, Carlsbad, CA, USA). cDNA was synthesised with Maxima reverse transcriptase and oligo (dT) primers (Thermo Fisher, Waltham, MA, USA). For all primer and probe details, see ESM Table 2. Relative cytokine expression was determined by real-time quantitative PCR (LightCycler 480; Roche, Basel, Switzerland). All cytokine signals were normalised to $\beta$-actin expression. Data are shown in relation to 4-week-old normal chow signal. The $p$ values are calculated from $\Delta \mathrm{C}_{\mathrm{t}}$ values $\left[\mathrm{C}_{\mathrm{t}}(\right.$ target $)-\mathrm{C}_{\mathrm{t}}($ reference $\left.)\right]$.

Western blot analysis of epithelial IL-18 expression For epithelial cell IL-18 immunoblot analysis the colon was emptied, rinsed with PBS and cut open longitudinally. The colon was agitated in $1 \mathrm{mmol} / 1$ EDTA-Hanks' balanced salt solution at $37^{\circ} \mathrm{C}$ for $30 \mathrm{~min}$. The solution was collected and centrifuged to pellet extracted cells. Cells were resuspended in $1 \mathrm{ml}$ RIPA buffer and lysed for $30 \mathrm{~min}$ at $4^{\circ} \mathrm{C}$. The lysate was centrifuged 
for $30 \mathrm{~min}$ at $14,000 \mathrm{~g}\left(4^{\circ} \mathrm{C}\right)$ to remove cell debris. Protein concentration was measured using the Bradford assay (Bio-Rad, Hercules, CA, USA). Western blot was performed on $10 \%$ SDS-PAGE gels and proteins were transferred onto PVDF membrane (Millipore, Darmstadt, Germany). Proteins IL-18 and actin were probed with monoclonal antibodies (anti-mouse IL-18, clone 39-3F from MBL, Nagoya, Japan; anti-actin, AC-40 from Sigma-Aldrich, St Louis, MO, USA) followed by second-step peroxidase-labelled anti-rat IgG (Cell Signaling Technology, Beverly, MA, USA). Immunoreactive bands were visualised on $\mathrm{x}$-ray films after reaction with ECL substrate (Thermo Fisher). The density of the IL-18 band was compared with that of the actin band from the same membrane. Band densities were measured using Multiskan EX photometer (Thermo Fisher).

Analysis of SCFAs and bacterial DNA in the large intestine Colon and caecum contents were pooled from three 7 -week-old mice $(n=15)$. Concentrations of SCFAs were measured by gas chromatography. Total bacterial DNA was isolated and stratified on the basis of guanosine and cytidine $(\mathrm{G}+\mathrm{C})$ nucleotide content. The total amount of bacteria was calculated as copy numbers per weight of content (Alimetrics, Espoo, Finland; www.alimetrics.com/, [12]).

DNA extraction for the next-generation sequencing analysis Total DNA was extracted from $100 \mathrm{mg}$ frozen, pooled (three mice) colon and caecum samples with GenoXtract Stool Extraction Kit VER 2.0 (Hain Lifescience, Nehren, Germany). The purity and concentration of the DNA samples were measured using a Nanodrop ND-1000 spectrophotometer (Thermo Fisher) at 230, 260 and $280 \mathrm{~nm}$.

Next-generation sequencing analysis for microbiota samples Next-generation sequencing was performed on an Illumina MiSeq system (Illumina, San Diego, CA, USA). For each genomic DNA sample, the V4 region of 16S rRNA gene (250 bp) was amplified by using Kapa HiFi PCR kit (Kapa Biosystems, Wilmington, MA, USA) and Veriti Thermal cycler (Thermo Fisher). The quality of libraries was confirmed by using Bioanalyzer 2100 (Agilent, Santa Clara, MA, USA) and the dataset was analysed by QIIME pipeline [21]. Data filtering was performed as described previously [22].

Statistical analyses Statistical analyses were carried out using GraphPad Prism 5 software (GraphPad, La Jolla, CA, USA). Statistical significance was determined using a two-tailed Student's $t$ test or one-way ANOVA with Dunnett's post hoc test. Test for survival was made with Gehan-BreslowWilcoxon test and test for trend with more than two groups with Mantel-Cox logrank test. A $p$ value of $<0.05$ was considered statistically significant.

\section{Results}

Supplementation of semisynthetic diets with FF promotes diabetes and insulitis We tested the effects of fibres on diabetogenesis using Basal mix 80\%-based semisynthetic diets in NOD mice. The basal mix was supplemented with milk proteins whey and casein and gluten, and either given as such or further supplemented with pectin and xylan (PX) or an equivalent amount (wt/wt) of cellulose (CE) (Fig. 1a). Compared with mice receiving normal laboratory chow (NC), there was a strong and significant decrease in disease incidence in mice fed the CE diet, whereas a milder decrease was observed in mice in the PX group. When compared with the group fed the semisynthetic diet without added fibre, the PX but not the CE group showed significantly decreased diabetes-free survival $(p=0.024)$. Importantly, the PX group showed decreased diabetes-free survival when compared with the CE group ( $p=0.049)$, suggesting that pectin and xylan, but not cellulose, have an effect on diabetogenesis. The groups showed varying mean survival and survival proportions: NC group, mean survival 17 weeks and 4.3\% disease free; PX group, mean survival 31 weeks and $47.1 \%$ disease free; $\mathrm{CE}$ group, mean survival remained undefined and $76.5 \%$ remained disease free (Fig. 1a). Statistical analysis showed a significant trend between groups with $\mathrm{NC}$ being the most diabetogenic and $\mathrm{CE}$ the least, while $\mathrm{PX}$ had a greater diabetogenic effect than $\mathrm{CE}(p<0.0001)$. Wheat gluten did not appear to promote diabetes incidence as the semisynthetic diet without added fibre contained 10\% wheat gluten and this group showed the best survival.

In addition, the severity of insulitis in the various diet groups was evaluated by grading islets according to lymphocyte infiltration (Fig. 1b, c). The CE diet significantly reduced insulitis when compared with $\mathrm{NC}(p=0.023)$, whereas the PX diet did not. There was also a significant difference between PX and CE diets with PX resulting in 1.8 times higher insulitis score than CE $(p=0.034)$. At 7 weeks of age, destruction of insulin-producing cells was not visible in islets of the $\mathrm{CE}$ group, but had begun in islets of both the PX and NC groups (Fig. 1d). These results suggest that pectin and xylan, but not cellulose, contribute to the development of autoimmune diabetes in NOD mice.

Expression of cytokines and epithelial stress-response genes in the colon depends on diet Analysis of mRNA from colon tissue showed higher transcript levels of the epithelial-stressrelated cytokine $I l 18$ in colons of 7-week-old mice fed the $\mathrm{NC}$ and PX diets when compared with the CE diet (Fig. 2a). Interestingly, the $\mathrm{NC}$ diet-fed mice showed profound $\mathrm{Il} 1 \mathrm{~b}$ expression in colons at 4 weeks of age, whereas $I l 1 b$ transcripts in PX and CE colons were barely detectable at this time (Fig. 2b). Together this lack of both $I l 1 b$ and $I l 18$ expression indicated reduction in proinflammatory activity in the colon of 

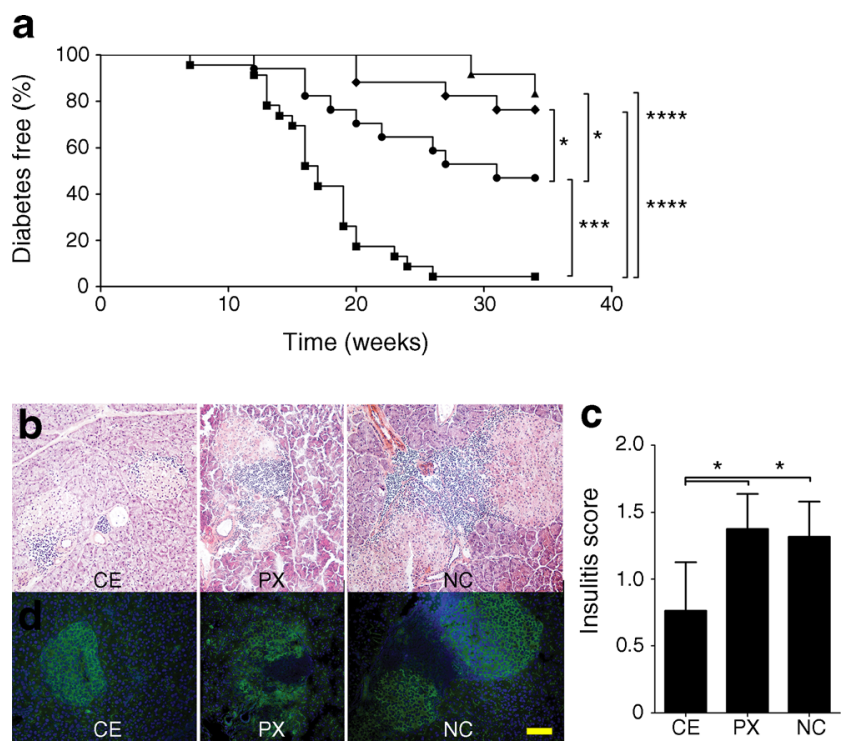

Fig. 1 FFs are diabetogenic in NOD mice. (a) Survival of mice fed diets made from Basal mix. Triangles, Basal mix with wheat gluten, whey and casein $(n=12)$; diamonds, Basal mix with whey, casein and cellulose added (CE; $n=17)$; circles, Basal mix with whey, casein, pectin and xylan added (PX; $n=17)$; squares, normal chow (NC; $n=23$ ). (b) Insulitis at 7 weeks in mice fed CE, PX and NC. (c) Insulitis score shows significant reduction in islet infiltration following CE diet ( $n=7$, errors bars show SD). (d) Insulin staining (green) shows no signs of beta cell destruction in islets of 7-weekold NOD mice on CE diet, while islets of mice on PX and NC diets already have clear areas with no insulin production. Nuclei stained with DAPI (blue). Scale bar, $200 \mu \mathrm{m} .{ }^{*} p<0.05,{ }^{* * *} p<0.001$ and ${ }^{* * * *} p<0.0001$

mice receiving semisynthetic diets supplemented with $\mathrm{CE}$ or $\mathrm{PX}$, with CE providing the strongest reduction.

Fig. $2 \mathrm{CE}$ and $\mathrm{PX}$ diets alter the levels of transcripts of the cytokine genes $I l 18$ (a), Illb (b), Il22 (c), Foxp3 (d), Chop (e), $\operatorname{Bip}(\mathbf{f}), \operatorname{Ror} \gamma t$ (g) and Il4 (h) in the large intestine of 4 - and 7-week-old NOD mice. $n=4$ ( $4 \mathrm{wk}, 4$ weeks) and $n=6$ (7 wk, 7 weeks), error shown as SEM, ${ }^{*} p<0.05,{ }^{* *} p<0.01$ and $* * * p<0.001$
Il22 was expressed in the colons of 4-week-old mice fed the CE and PX diets, whereas colons of the NC group showed very low expression levels. The CE diet induced a 20-fold $(p=0.039)$ increase and the PX diet induced a tenfold $(p=$ 0.008 ) increase in $I l 22$ expression when compared with the NC diet (Fig. 2c). Expression of Foxp3 was greatly upregulated in colons of the $\mathrm{CE}$ group at 4 weeks (5.5-fold, $p=0.033$ ) when compared with NC (Fig. 2d). The PX diet did not induce Foxp3 expression in the colon at all. Expression of epithelial stress-response genes Chop (also known as Ddit3) and Bip (also known as Hspa5) was significantly higher in mice receiving the $\mathrm{NC}$ diet at 4 weeks of age, as compared with mice receiving the $\mathrm{CE}$ or $\mathrm{PX}$ diets (Fig. 2e, f).

Further, mRNA expression of transcription factor Ror $\gamma t$ (also known as Rorc) showed a significant decrease with semisynthetic diets when compared with NC. At 4 weeks this effect was not yet statistically significant, but reached significant difference by 7 weeks (Fig. $2 \mathrm{~g}$ ). At 7 weeks, the colons of mice fed the CE diet showed a sevenfold $(p=0.004)$ decrease in Ror $\gamma t$ expression when compared with NC colons (Fig. 2g) and those of the mice fed the PX diet showed a threefold $(p=0.002)$ decrease. Importantly the CE diet was significantly more effective than PX in reducing the expression of Ror $\gamma t$. PX diet-induced Ror $\gamma t$ expression remained 2.3-fold higher than CE dietinduced Ror $\gamma t$ expression $(p=0.033)$. Also, we observed significantly increased Il4 expression in colons of the CE group (17.5-fold) at 4 weeks when compared with the NC group ( $p=0.036$, Fig. 2h).
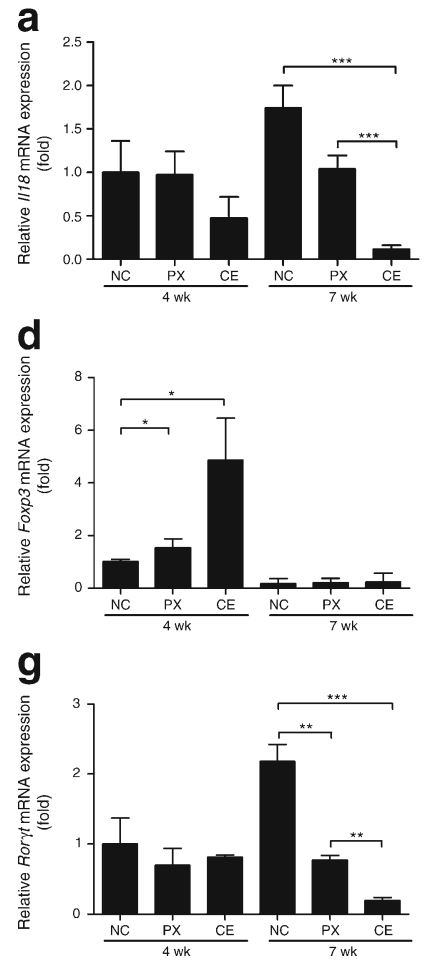

b
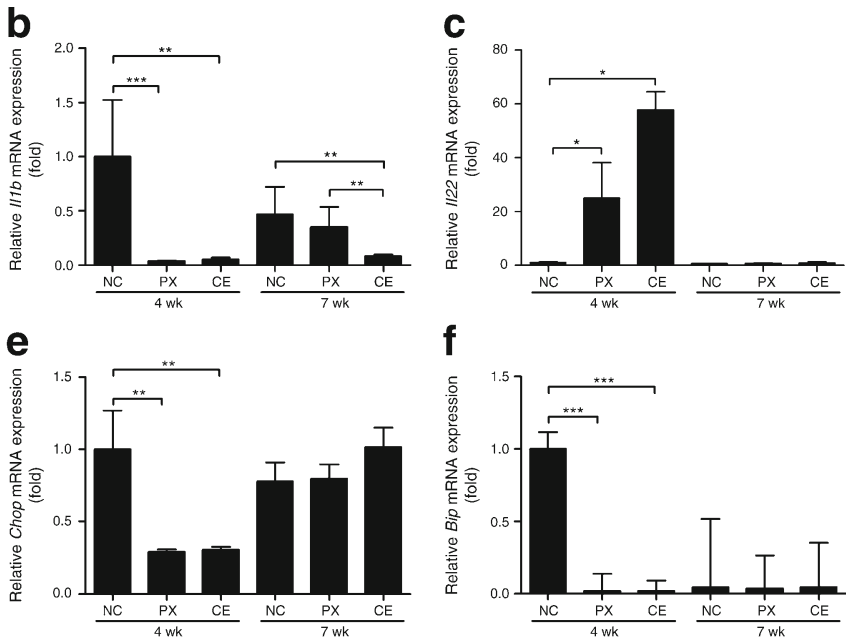

h

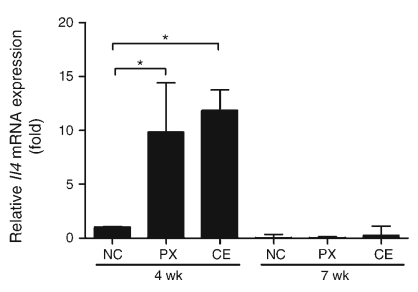


FF-free diet promotes Foxp3 and Tgfß transcripts in both mesenteric and pancreatic lymph nodes Analysis of PaLN and mesenteric lymph node (MLN) mRNA showed that both PX and CE diets induced expression of $T g f \beta$, regulatory T cell (Treg)-associated Foxp3 and Il4 in the PaLN (Fig. 3a-c). Importantly, in the gut-draining MLN, the CE diet induced $T g f \beta$ and Foxp3 significantly more than the PX diet. In MLNs the CE diet induced levels of Tgf $\beta$ expression 2.5-fold higher than those induced by the $\mathrm{NC}$ diet and 2.2-fold higher than those induced by the PX diet $(p=0.005)$ (Fig. 3a). CE diet induced Foxp3 expression 4.1-fold $(p=0.0003)$ in MLNs compared with NC and 1.9-fold ( $p=0.044)$ compared with PX diet (Fig. 3b). Expression of Il4 in MLNs was not affected by diet (Fig. 3c). Expression of If $n \gamma$, in either PaLNs or MLNs, was not significantly affected by diet (Fig. 3d).

FF-free diet reduces crypt length and epithelial IL-18 in the colon After 1 week on semisynthetic diets, no differences in colon histology were seen. After 4 weeks on diets, we observed 20\% thicker colon crypt epithelium in mice fed with NC compared with mice fed with CE or PX diets (Fig. 4a, c). We isolated epithelial cells from the colon and analysed levels of bioactive IL-18 protein by western blot. In line with mRNA analysis, active IL-18 was expressed at significantly higher levels in PX colons than in CE colons $(p=0.0004)$, while NC colon samples showed the most intensive epithelial IL-18 protein expression (Fig. 4b, d).

Effect of FF on GM composition Profiling total bacterial DNA on the basis of $\mathrm{C}+\mathrm{G}$ fractionating provides an amplification step-independent readout of microbiota composition. Interestingly, different diets led to different large-intestine bacterial composition, with NC harbouring the largest fraction
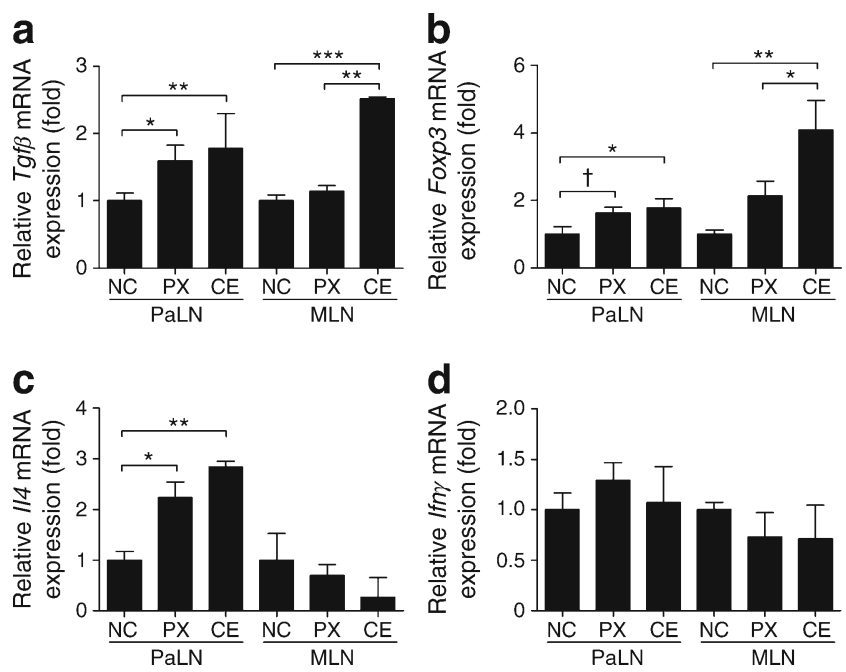

Fig. $3 \mathrm{CE}$ and PX diets alter the levels of transcripts of immunoregulatory genes $\operatorname{Tgf} \beta$ (a), Foxp3 (b), Il4 (c) and Ifn $\gamma(\mathbf{d})$ in PaLNs and MLNs $\left(n=6\right.$, error bars show SEM, ${ }^{*} p<0.05,{ }^{*} p<0.01$ and $\left.* * * p<0.001\right)$; ${ }^{\dagger} p=0.059$
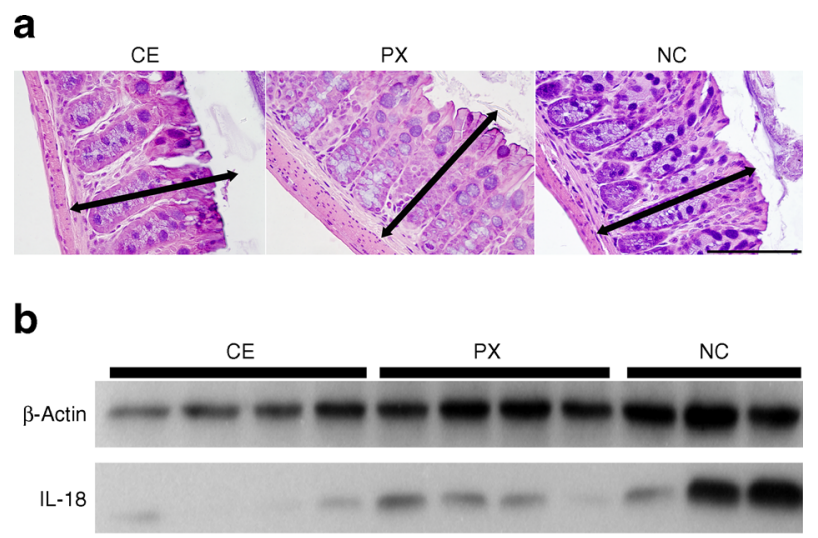

C

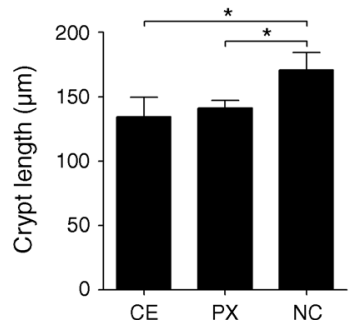

d

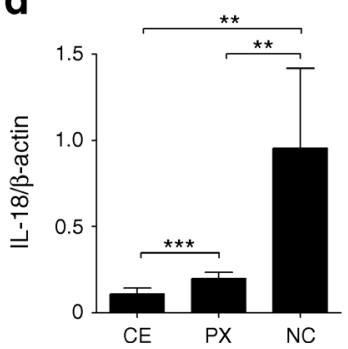

Fig. 4 Reduced crypt length and epithelial IL-18 expression in mice fed semisynthetic diets. (a) Representative sections of colon tissue showing differences in epithelial crypt length. Arrows represent crypt length in mice fed NC. Scale bar, $100 \mu \mathrm{m}$. (b) Representative western blot showing IL-18 expression in comparison with $\beta$-actin. (c) Quantification of decrease in colon mucosa thickness following PX and CE diet $(n=4$, errors bars show SD). (d) Quantification of the signal in immunoblot bands by densitometry analysis shows significant differences between diets $(n=7$, error bars show SEM, ${ }^{*} p<0.05,{ }^{* *} p<0.01$ and ${ }^{* * *} p<0.001$ )

of low $\mathrm{G}+\mathrm{C}(30-37 \%)$ bacteria when compared with other diets (Fig. 5). Low $\mathrm{G}+\mathrm{C}$ bacteria include, for example, the Gram-positive members of the Firmicutes phylum from the Bacilli lineage and Mollicutes. Low $\mathrm{G}+\mathrm{C}$ bacteria were reduced with PX and $\mathrm{CE}$ diets and at the same time enrichment of other $\mathrm{G}+\mathrm{C}$ fractions was observed (Fig. 5a). Mice on the PX diet had significant enrichment of $\mathrm{G}+\mathrm{C}$ fractions (43-48\%) while mice on the $\mathrm{CE}$ diet had profound enrichment of high $\mathrm{G}+\mathrm{C}$ fractions (55-67\%) when compared with either the PX or NC diets. High $\mathrm{G}+\mathrm{C}$ bacteria include members of the Actinobacteria phylum, which are known to pose effects beneficial to their host's health. Similarly when compared with other diets, PX showed significant enrichment in the $\mathrm{G}+\mathrm{C}$ area (44-46\%), including the Lactobacillus as well as various Gram-negative Bacteroides species. Both PX and CE diets resulted in a slight but significant reduction in total bacterial load (Fig. 5b).

We further analysed whole microbiomes from largeintestine samples by $16 \mathrm{~S}$ rRNA sequencing and identified $12,701,908$ reads for the 14 samples, with an average of 907,279 reads per sample (SD 284,920; minimum 610,532, maximum 1,682,805). Changing the diet from $\mathrm{NC}$ to $\mathrm{CE}$ not only decreased the total bacterial load but also significantly 


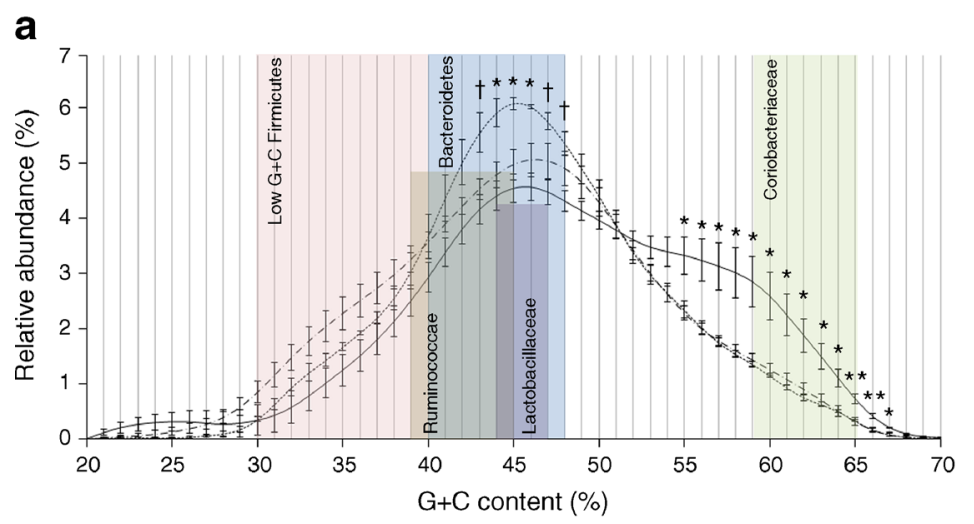

b

C
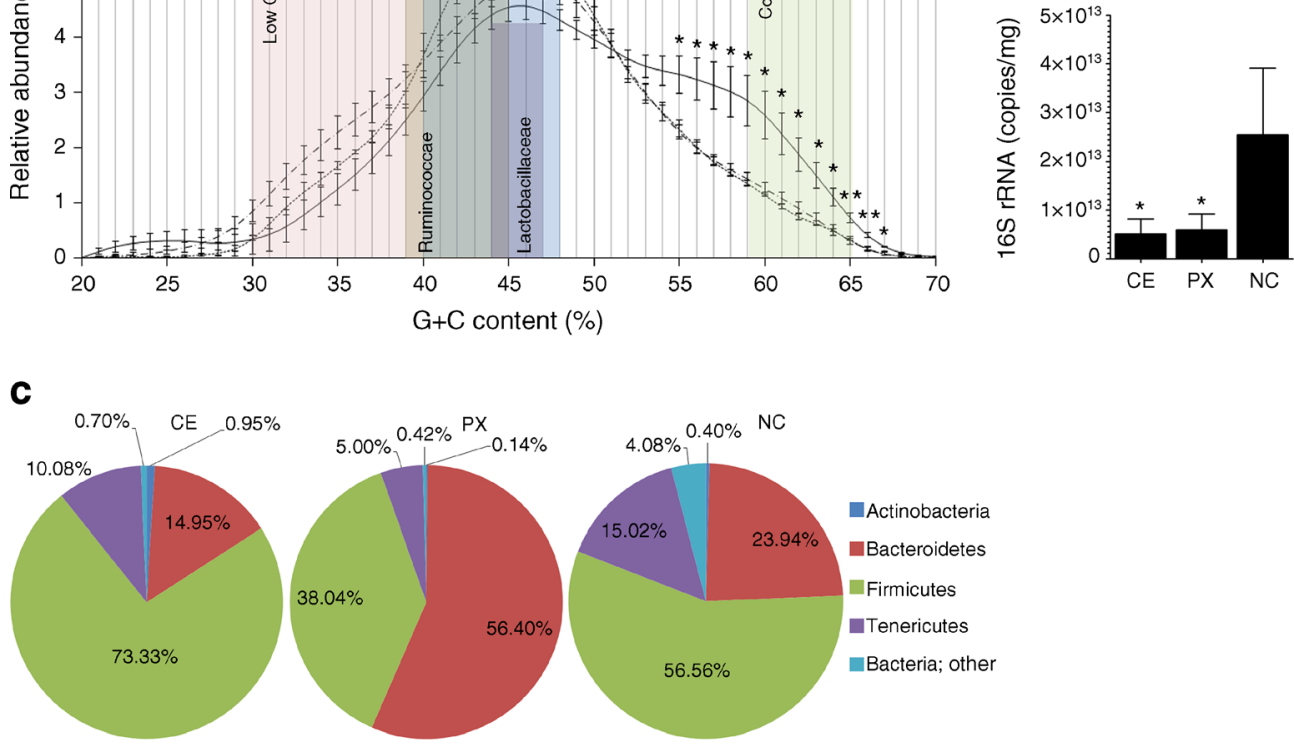

d

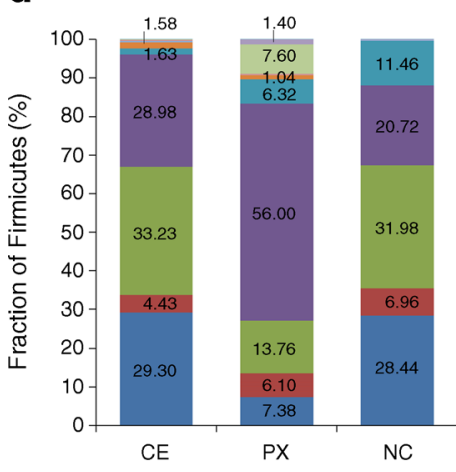

e

f

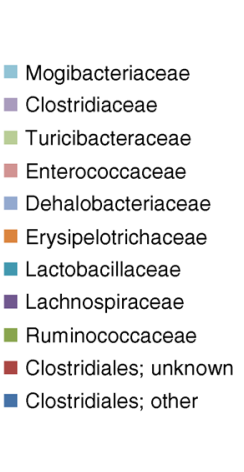

NC3

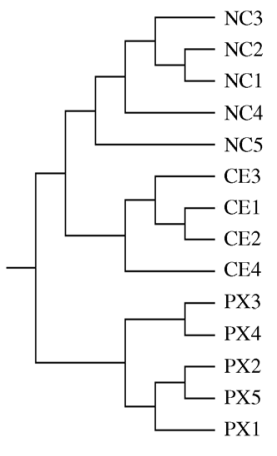

g
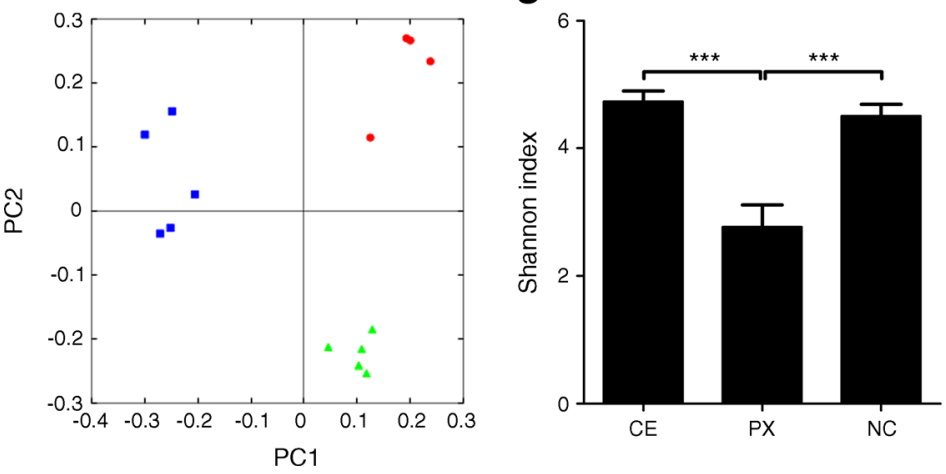

increased Firmicutes and simultaneously decreased Bacteroidetes. Addition of pectin and xylan had opposing effects since the PX diet significantly increased Bacteroidetes while decreasing Firmicutes. Tenericutes were decreased by both the CE and PX diets compared with the NC diet (Fig. 5c). The diet increased most noticeably the Gram-positive Ruminococcaceae and Coriobacteriaceae families. Ruminococcaceae are common bacteria able to ferment 
Fig. 5 Impact of FFs on microbiota. (a) CE diet free from FFs alters the $\mathrm{G}+\mathrm{C}$ profile of GM dramatically, while addition of pectin and xylan to this diet normalises this profile considerably. $\mathrm{CE}$ diet enriches colonic bacteria with $\mathrm{G}+\mathrm{C}$ content $55-67 \%$, while PX diet allows for significant enrichment of bacteria with genome $\mathrm{G}+\mathrm{C}$ content $43-48 \%$ (solid line, $\mathrm{CE}$; dashed line, $\mathrm{PX}$; dotted line, $\mathrm{NC} ; n=15$ mice/group, errors shown as $\mathrm{SD},{ }^{*} p<0.05,{ }^{* *} p<0.01$ and ${ }^{* * *} p<0.001$ vs NC; ${ }^{\dagger} p<0.05$ PX vs CE). Selected bacterial clusters with defined $\mathrm{G}+\mathrm{C}$ content are shown in the coloured areas. (b) Total bacterial load is decreased by semisynthetic diets ( $n=15$ mice/group, errors shown as $\mathrm{SD},{ }^{*} p<0.05$ ). (c) Semisynthetic diets with different fibre composition change GM differentially on the level of major phyla. PX diet reverses Bacteroidetes to Firmicutes ratio ( $n=5$ mice/group). (d) Comparison of composition within Firmicutes. Note the enrichment of mucin-degrading Ruminococcaceae following diabetes-protective $\mathrm{CE}$ diet (combining the proportions in $\mathbf{c}$ and $\mathbf{d}$, shown in dark green). (e) NC, CE and PX mice (1-5) cluster to form distinct GM compositions. (f) Principal component analysis of microbiota with CE (red circles, $n=4$ ), PX (blue squares, $n=5$ ) and NC (green triangles, $n=5$ ) diets. Principal components PC1 and PC2 explain $21.52 \%$ of the total variance (PC1 $11.1 \%$ and PC2 10.41\%). (g) Total microbiota diversity in mice as measured by the Shannon index, $* * * p<0.001$

cellulose (reviewed in [23]). This increase was not observed in mice on the PX diet. In contrast, the Ruminococcaceae fraction was decreased and simultaneously a profound enrichment of Bacteroidetes was observed in the PX group (see Table 1 for summary of $p$ values). The microbiomes of each diet clearly differed from one another as shown by dendrogram as well as by principal component analysis (Fig. 5e, f). Furthermore, the PX diet significantly reduced the diversity of microbiota, as demonstrated by differences in the Shannon index (Fig. 5g).

Table 1 Summary of $p$ values describing differences in microbiota between diets

\begin{tabular}{lllll}
\hline Phylum & $p$ value & CE vs PX & CE vs NC & PX vs NC \\
\hline Actinobacteria & 0.0034 & $*$ & $* *$ & NS \\
Bacteroidetes & $<0.0001$ & $* * *$ & $\mathrm{NS}$ & $* * *$ \\
Tenericutes & 0.0499 & $\mathrm{NS}$ & $\mathrm{NS}$ & $*$ \\
Firmicutes & $<0.0001$ & $* * *$ & $*$ & $* *$ \\
Mogibacteriaceae & 0.0004 & $* * *$ & $\mathrm{NS}$ & $*$ \\
Clostridiaceae & 0.14 & $\mathrm{NS}$ & $\mathrm{NS}$ & $\mathrm{NS}$ \\
Turicibacteraceae & 0.0035 & $* *$ & $\mathrm{NS}$ & $* *$ \\
Enterococcaceae & 0.11 & $\mathrm{NS}$ & $\mathrm{NS}$ & $\mathrm{NS}$ \\
Dehalobacteriaceae & 0.065 & $\mathrm{NS}$ & $\mathrm{NS}$ & $\mathrm{NS}$ \\
Erysipelotrichaceae & 0.069 & $\mathrm{NS}$ & $\mathrm{NS}$ & $\mathrm{NS}$ \\
Lactobacillaceae & 0.016 & $\mathrm{NS}$ & $*$ & $\mathrm{NS}$ \\
Lachnospiraceae & $<0.0001$ & $* * *$ & $\mathrm{NS}$ & $* * *$ \\
Ruminococcaceae & 0.0006 & $* *$ & $\mathrm{NS}$ & $* *$ \\
Clostridiales; unknown & 0.56 & $\mathrm{NS}$ & $\mathrm{NS}$ & $\mathrm{NS}$ \\
Clostridiales; other & 0.0003 & $* * *$ & $\mathrm{NS}$ & $* * *$ \\
\hline
\end{tabular}

$p$ value determined by one-way ANOVA, from analysis of all three groups

${ }^{*} p<0.05,{ }^{* *} p<0.01, * * * p<0.001$
Effect of diets on metabolic products in the large intestine There were significant differences in the concentration of SCFAs between the diets (Fig. 6). The CE diet reduced the amount of total SCFAs very strongly while the PX diet reduced total SCFAs significantly less, when compared with the NC diet. Interestingly, the PX diet increased the production of propionic acid, even when compared with the NC diet. Importantly, the PX diet differs from the CE diet only by fibre composition, indicating differential effects between pectin and xylan when compared with cellulose.

\section{Discussion}

In this study, we show evidence for the first time that FFs in the diet associate with development of autoimmune diabetes. Our data indicate that the addition of two such fibres, pectin and xylan, to semisynthetic FF-free diet, significantly counteracts its diabetes-preventing effect in NOD mice. It is generally known that semisynthetic diets are diabetes-retardant both in NOD mice and BB rats and, apart from their usefulness in modifying protein composition in the diet, they are free from FF [24]. a

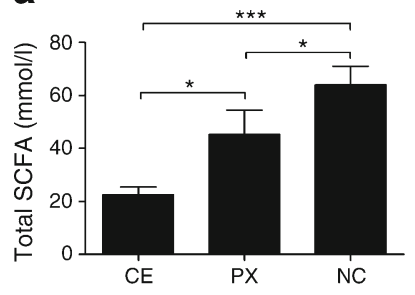

C
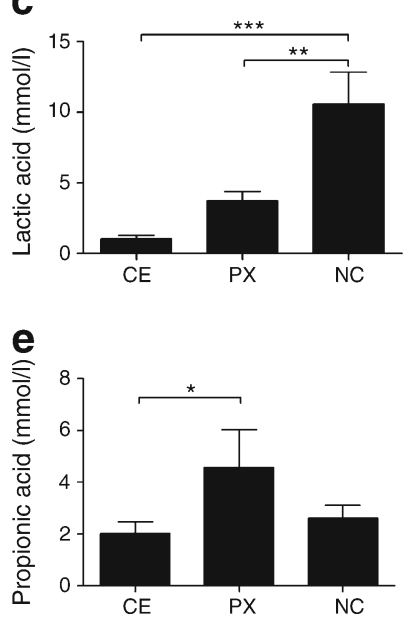

b

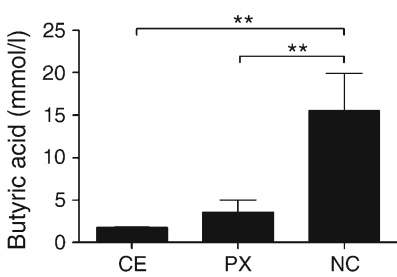

d

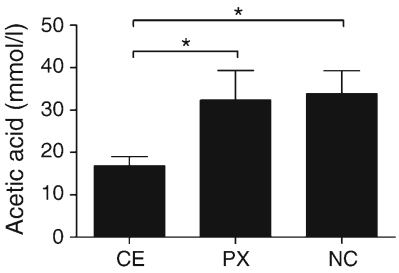

Fig. 6 Concentration of SCFA metabolites of FFs in the colon and the effect of diets containing different fibres. (a) Total short-chain fatty acid. (b) Butyric acid. (c) Lactic acid. (d) Acetic acid. (e) Propionic acid. $n=15$ mice/group, errors shown as $\mathrm{SD},{ }^{*} p<0.05,{ }^{*} p<0.01,{ }^{* * *} p<0.001$ 
Dietary proteins are important both in human type 1 diabetes $[25,26]$ and in animal models of autoimmune diabetes $[9,27]$. However, recent evidence also points towards a role for GM in type 1 diabetes [24, 28]. Many fibres have prebiotic effects, and are fermented by GM [29]. Although subject to culturing conditions and culture media used in each study, Chassard et al reported isolation of six different Bacteroides $\mathrm{sp}$. in culturing conditions in which xylan was used as the sole energy source [30]. This suggests a prominent role for Bacteroides as xylan-degrading organisms. Also, pectin is efficiently degraded by Bacteroides sp. and Eubacterium eligens [31]. In human studies, the potential association between Bacteroidetes-rich microbiota and progression to islet autoimmunity $[24,28,32,33]$ warrants consideration of these fibres in the diet of individuals at risk of developing type 1 diabetes. Our data revealed that when compared with a semisynthetic diet free from FFs, standard laboratory chow associates with deeper crypts, higher levels of proinflammatory and stress-related transcripts and epithelial IL-18 expression in the colon, and that part of this appears to be ascribed to FFs in the standard diet. Considering the effects of colon irritation on T cell activation in PaLNs [14] and increased crypts in colitis models [34], our data emphasise the potential relevance of dietary fibres and colon irritation in the pathogenesis of autoimmune diabetes, as modelled in Fig. 7. The proposed model incorporates the effects of our diets and related microbiota on gut homeostasis in the colon, and their potential link to proinflammatory conditioning of immune responses in PaLNs [14]. According to our model, FFs can not only support gut homeostasis by providing beneficial metabolites for intestinal epithelial cells [35] but also promote outgrowth of certain bacteria (e.g. Bacteroidetes), which could induce stress to gut epithelium and activate inflammatory responses. A diabetes-retardant diet free from FFs associated with microbiota rich in Firmicutes may induce Tregs [36] with anti-inflammatory activity. The balance of pro- and antiinflammatory signals in the colon is ultimately reflected in PaLNs [14], where it could impact on the initiation or progression of autoimmune diabetes in predisposed individuals.

Our whole microbiome data demonstrated that inclusion of pectin and xylan in the rodent diet significantly increases the presence of Bacteroides and at the phylum level reverses the Bacteroidetes to Firmicutes ratio from 0.2 to 1.5 , indicating a 7.5-fold increase in Bacteroidetes over Firmicutes. As previous studies in rodents and humans have suggested that predominance of Bacteroides associates with type 1 diabetes development $[24,28,32,33]$, the observed increase in Bacteroides induced by pectin and xylan further suggests a microbiota-mediated effect on diabetogenesis. Our 16S RNA gene sequencing results show that the NOD mice used in this study are devoid of known mucin-degrading bacteria such as Verrucomicrobiales and Prevotellaceae. These genera have been associated with protection from diabetes [28]. Another mucin-degrading genus occupying the same environmental niche as Verrucomicrobiales (reviewed in [37]), namely the Ruminococcaceae, was enriched by the diabetes-protective CE diet. These results support the notion that mucindegrading bacteria may counteract the development of autoimmune diabetes.

FFs are numerous and many of them are either constituents of roots, berries and vegetables or alimentary products of the modern food industry, ingested daily as part of the normal diet. In this study, we found that two such fibres, pectin and xylan, impact strongly on GM and condition the colon towards creating the environment observed in NOD mice fed standard, diabetogenic, laboratory chow. In light of the present study, it appears that previously observed associations between microbiota changes and type 1 diabetes-related

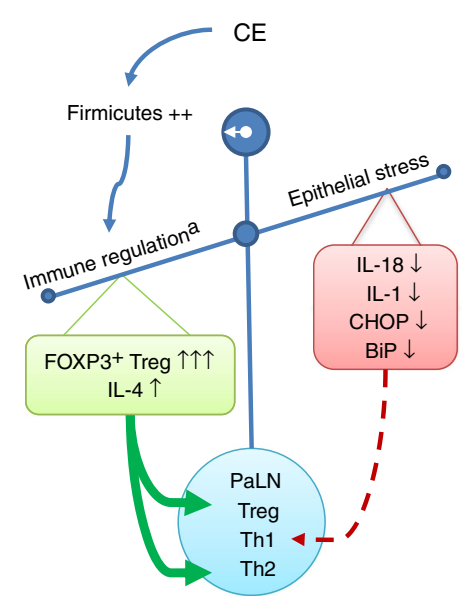

Fig. 7 Dietary fibres differentially affect the gut immune regulation and epithelial stress. CE diet induces immune regulation, probably due to enrichment of specific clostridial species ('ref. [36]). PX diet induces immune regulation by propionate production ('ref. [35]) but also increases gut epithelial stress. Diet can induce changes to microbiota
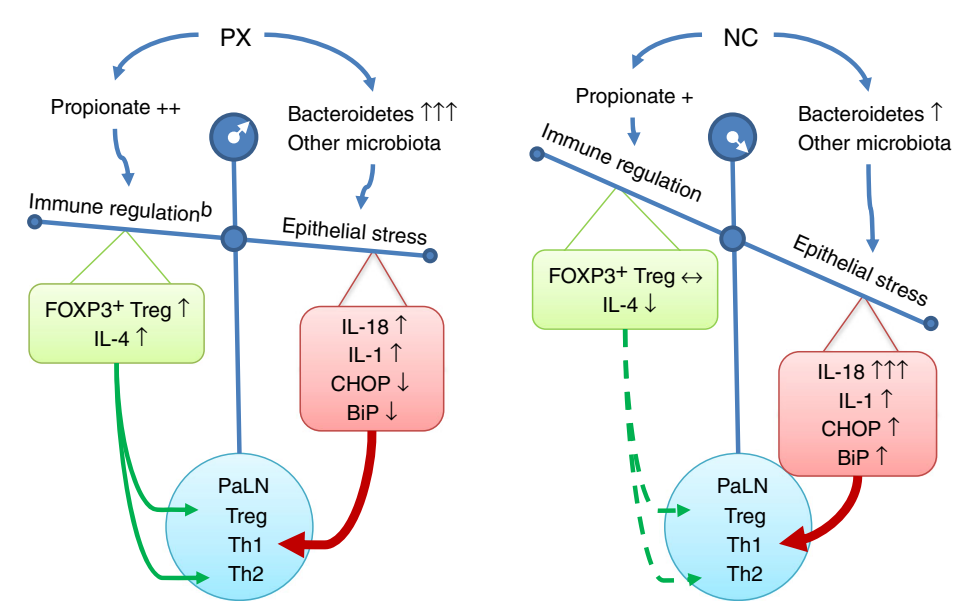

balance and tip the scale either way. The effects are carried over to the PaLNs where disruption of immune regulation can facilitate the progress of autoimmune diabetes [14]. FOXP3, forkhead box P3; Th1, T helper 1 cell; Th2, T helper 2 cell 
autoimmunity may in part be explained by individual differences in ingestion of these fibres and their effects on GM.

Acknowledgements The authors thank M. Jaakkola and L. Lauren (Medical Microbiology and Immunology, University of Turku) for their technical contributions to experiments, as well as A. Demasör and R. Mankonen for animal care at the Turku University central animal core.

Funding This study was supported by Päivikki and Sakari Sohlberg Foundation, Diabetes Research Foundation Finland, Jalmari and Rauha Ahokas Foundation, Emil Aaltonen Foundation, Turku University Foundation and the Academy of Finland.

Duality of interest The authors declare that there is no duality of interest associated with this manuscript.

Contribution statement All authors made substantial contributions to the conception and design of the study, acquisition of data or analysis and interpretation of data and drafted the article or revised it critically for important intellectual content. All authors gave final approval of the version to be published. RT and AH are responsible for the integrity of the work as a whole.

\section{References}

1. Gallegos AM, Bevan MJ (2004) Driven to autoimmunity: the nod mouse. Cell 117:149-151

2. Anderson MS, Bluestone JA (2005) The NOD mouse: a model of immune dysregulation. Annu Rev Immunol 23:447-485

3. Savilahti E, Ormala T, Saukkonen T et al (1999) Jejuna of patients with insulin-dependent diabetes mellitus (IDDM) show signs of immune activation. Clin Exp Immunol 116:70-77

4. Auricchio R, Paparo F, Maglio M et al (2004) In vitro-deranged intestinal immune response to gliadin in type 1 diabetes. Diabetes 53:1680-1683

5. Tiittanen M, Westerholm-Ormio M, Verkasalo M, Savilahti E, Vaarala O (2008) Infiltration of forkhead box P3-expressing cells in small intestinal mucosa in coeliac disease but not in type 1 diabetes. Clin Exp Immunol 152:498-507

6. Hanninen A, Salmi M, Simell O, Jalkanen S (1996) Mucosaassociated (beta 7-integrinhigh) lymphocytes accumulate early in the pancreas of NOD mice and show aberrant recirculation behavior. Diabetes 45:1173-1180

7. Hanninen A, Jaakkola I, Jalkanen S (1998) Mucosal addressin is required for the development of diabetes in nonobese diabetic mice. J Immunol 160:6018-6025

8. Bosi E, Molteni L, Radaelli MG et al (2006) Increased intestinal permeability precedes clinical onset of type 1 diabetes. Diabetologia 49:2824-2827

9. Maurano F, Mazzarella G, Luongo D et al (2005) Small intestinal enteropathy in non-obese diabetic mice fed a diet containing wheat. Diabetologia 48:931-937

10. Atkinson MA, Winter WE, Skordis N, Beppu H, Riley WM, Maclaren NK (1988) Dietary protein restriction reduces the frequency and delays the onset of insulin dependent diabetes in BB rats. Autoimmunity 2:11-19

11. Beales PE, Elliott RB, Flohe S et al (2002) A multi-centre, blinded international trial of the effect of $\mathrm{A}(1)$ and $\mathrm{A}(2)$ beta-casein variants on diabetes incidence in two rodent models of spontaneous type I diabetes. Diabetologia 45:1240-1246
12. Emani R, Asghar MN, Toivonen R et al (2013) Casein hydrolysate diet controls intestinal $\mathrm{T}$ cell activation, free radical production and microbial colonisation in NOD mice. Diabetologia 56:1781-1791

13. Brugman S, Klatter FA, Visser JT et al (2006) Antibiotic treatment partially protects against type 1 diabetes in the Bio-Breeding diabetes-prone rat. Is the gut flora involved in the development of type 1 diabetes? Diabetologia 49:2105-2108

14. Turley SJ, Lee JW, Dutton-Swain N, Mathis D, Benoist C (2005) Endocrine self and gut non-self intersect in the pancreatic lymph nodes. Proc Natl Acad Sci U S A 102:17729-17733

15. Lee AS, Gibson DL, Zhang Y, Sham HP, Vallance BA, Dutz JP (2010) Gut barrier disruption by an enteric bacterial pathogen accelerates insulitis in NOD mice. Diabetologia 53:741-748

16. Alam C, Valkonen S, Palagani V, Jalava J, Eerola E, Hanninen A (2010) Inflammatory tendencies and overproduction of IL-17 in the colon of young NOD mice are counteracted with diet change. Diabetes 59:2237-2246

17. Vanhoutvin SA, Troost FJ, Kilkens TO et al (2009) The effects of butyrate enemas on visceral perception in healthy volunteers. Neurogastroenterol Motil 21:952-e76

18. Hamer HM, de Preter V, Windey K, Verbeke K (2012) Functional analysis of colonic bacterial metabolism: relevant to health? Am J Physiol Gastrointest Liver Physiol 302:G1-G9

19. Borthakur A, Bhattacharyya S, Dudeja PK, Tobacman JK (2007) Carrageenan induces interleukin- 8 production through distinct Bcl10 pathway in normal human colonic epithelial cells. Am J Physiol Gastrointest Liver Physiol 292:G829-G838

20. Bhattacharyya S, Borthakur A, Dudeja PK, Tobacman JK (2008) Carrageenan induces cell cycle arrest in human intestinal epithelial cells in vitro. J Nutr 138:469-475

21. Caporaso JG, Kuczynski J, Stombaugh J et al (2010) QIIME allows analysis of high-throughput community sequencing data. Nat Methods 7:335-336

22. Bokulich NA, Subramanian S, Faith JJ et al (2013) Quality-filtering vastly improves diversity estimates from Illumina amplicon sequencing. Nat Methods 10:57-59

23. Russell JB, Rychlik JL (2001) Factors that alter rumen microbial ecology. Science 292:1119-1122

24. de Goffau MC, Luopajarvi K, Knip M et al (2013) Fecal microbiota composition differs between children with beta-cell autoimmunity and those without. Diabetes 62:1238-1244

25. Norris JM, Barriga K, Klingensmith G et al (2003) Timing of initial cereal exposure in infancy and risk of islet autoimmunity. JAMA 290: $1713-1720$

26. Knip M, Virtanen SM, Seppa K et al (2010) Dietary intervention in infancy and later signs of beta-cell autoimmunity. N Engl J Med 363: 1900-1908

27. Elliott RB, Reddy SN, Bibby NJ, Kida K (1988) Dietary prevention of diabetes in the non-obese diabetic mouse. Diabetologia 31:62-64

28. Brown CT, Davis-Richardson AG, Giongo A et al (2011) Gut microbiome metagenomics analysis suggests a functional model for the development of autoimmunity for type 1 diabetes. PLoS One 6:e25792

29. Flint HJ, Bayer EA, Rincon MT, Lamed R, White BA (2008) Polysaccharide utilization by gut bacteria: potential for new insights from genomic analysis. Nat Rev Microbiol 6:121-131

30. Chassard C, Delmas E, Lawson PA, Bernalier-Donadille A (2008) Bacteroides xylanisolvens sp. nov., a xylan-degrading bacterium isolated from human faeces. Int J Syst Evol Microbiol 58:1008-1013

31. Salyers AA, West SE, Vercellotti JR, Wilkins TD (1977) Fermentation of mucins and plant polysaccharides by anaerobic bacteria from the human colon. Appl Environ Microbiol 34:529-533

32. Roesch LF, Lorca GL, Casella G et al (2009) Culture-independent identification of gut bacteria correlated with the onset of diabetes in a rat model. ISME J 3:536-548

33. Giongo A, Gano KA, Crabb DB et al (2011) Toward defining the autoimmune microbiome for type 1 diabetes. ISME J 5:82-91 
34. Alenghat T, Osborne LC, Saenz SA et al (2013) Histone deacetylase 3 coordinates commensal-bacteria-dependent intestinal homeostasis. Nature 504:153-157

35. Smith PM, Howitt MR, Panikov N et al (2013) The microbial metabolites, short-chain fatty acids, regulate colonic Treg cell homeostasis. Science 341:569-573
36. Atarashi K, Tanoue T, Shima $\mathrm{T}$ et al (2011) Induction of colonic regulatory $\mathrm{T}$ cells by indigenous Clostridium species. Science 331: $337-341$

37. Ouwerkerk JP, de Vos WM, Belzer C (2013) Glycobiome: bacteria and mucus at the epithelial interface. Best Pract Res Clin Gastroenterol $27: 25-38$ 